\title{
Photonics for MS study in radiocommunications
}

\section{Rudolf Volner, Milos Klima, Dasa Ticha}

Rudolf Volner, Milos Klima, Dasa Ticha, "Photonics for MS study in radiocommunications," Proc. SPIE 4588, Seventh International Conference on Education and Training in Optics and Photonics, (28 May 2002); doi: $10.1117 / 12.468744$

SPIE Event: Education and Training in Optics and Photonics 2001, 2001, Singapore, Singapore 


\title{
Photonics for MS study in Radiocommunications
}

\author{
Rudolf Volner, ${ }^{1}$, Milos Klima, ${ }^{2}{ }^{* *}$, Dasa Ticha, ${ }^{* 3}$ \\ Dept. of Telecommunications, ${ }^{* *}$ Dept. Of Radioelectronics
}

\begin{abstract}
The paper is devoted to an education of Photonics at the Dept. of Telecommunications, Faculty of Electrical Engineering, at the University of Zilina. Originated from the university historical development the photonic subjects are implemented in two basic areas: Telecommunication Technology and Radiocommunication Technology. From the school year 1994/95 the new subject Photonics has been taught ad it has attracted numerous students. The subject is focused on both physical principles and system application. The relevant parts can be listed as: interaction photon - matter, photonic receivers and transmitters, modulation and demodulation in Photonics, photonic networks - narrowband and wideband, photonic switches, image sensors and displays.

The education of Photonics has been supported by research activities in the field of applied photonic system for signal (data) transmission and selected results have been implemented into the subject structure. The paper listed a detailed content of the subject in two fields: lectures and experimental laboratory excercises. As an integral part of the course we plan to implement selected experiments from the area of 2D photonic (image) processing and to expand the imaging photonic part.
\end{abstract}

Keywords: Photonics, education

\section{INTRODUCTION}

It is widely discussed that the future of communication world will be in two main directions. All communications between fixed points will be done by the fiber optics, while based upon this backbone infrastructure there will be the second world of radio and infrared communications applied by mobile or portable devices. The radio and fiber are dual technologies: the radio provides mobility to the end user but has severe bandwidth limitations, the fiber has severely limited deployment but brings enormous bandwidth potential to the point it reaches [1].

Optoelectronic technology and optical networking will become the key means of future communications infrastructure causing an elimination of the several traditional electromagnetic signal based communications. The electromagnetic signals carried by copper (or coaxial) cables suffer an attenuation and are subjected to errors due to noise and moreover such systems have limited data rates. The full impacts of essentially extremely large bandwidth and low loss rates are only beginning and they will dramatically reshape the computing technologies of the future of our networks, processors and applications. While in the past, the communication link was a bottleneck, this link now holds the potential to become the technology of new modes of computing far beyond those existing today.

\footnotetext{
${ }^{1}$ MSEE, PhD, Assoc. Professor, volner@zoznam.sk,

${ }^{2}$ MSEE, PhD, Professor, klima@feld.cvut.cz , Dept. Of Radioelectronics, Faculty of Electrical Engineering, Czech Technical University in Prague, Czech Republic

${ }^{3}$ MSEE, PhD, Assist. Professor, ticha@fel.utc.sk, Dept. of Telecommunications, Faculty of Electrical Engineering, University of Zilina, Slovak Republic,
} 


\section{EDUCATION PHOTONICS}

Nowadays the Department of Telecommunications, Technical Univerzity in Zilina, participates in an education in two directions:

- Telecommunication technology

- Radiocommunication technology

The latest development in the world demonstrates the requirements to implement the Photonics disciplines into the Radiocommunications. In the school year 1994/1995 a new subject Photonics was established to attract student attention to the following topics:

- interaction between photon and atom,

- photonic receivers and transmitters,

- optical waveguides,

- modulating and demodulating systems in photonics,

- photonic networks - narrowband and wideband,

- photonic switches,

- image sensors and displays.

The education in this subject is supported by a research in the field of photonic system exploitation for the signal transmission. The educational curricula follows the content of our textbook [2]. The research results have been published on both Slovak and international conferences.

The next generation photonic transmission system has three basic elements:

- the photonic source,

- the transmission medium,

- the detector.

Based upon this example, one can easily identify the photonic telecommunication system components:

- $\quad$ photonic source - infrared light - emitting diode (LED) or injection laser diode (ILD),

- optical fiber - single mode or multimode fiber,

- amplifier - electronic repeater or erbium-doped optical amplifier,

- photonic detector - photodiode and photo transistor.

The source and end-system devices are typically telephones, computers, video cameras or TVs. 
There are three classes of all-optical networks differing in the topology. The simplest one, a typical WDM point - to - point link, consists of several wavelength-differentiated distributed feedback (DFB) lasers at the transmitting ends of different wavelength channels and photodetectors at the receiving ends with a passive MUX/DEMUX connecting the two ends of particular link.

Broadcast-and-select networks are based upon a passive star coupler device connected to several stations in a star topology. This device is a piece of glass that splits the signal from any of its ports to all ports. As a result, it offers an optical equivalent to radio systems - each transmitter broadcasts its signal on a different wavelength, and the receivers can be tuned to receive the relevant signal. The main networking challenge in such networks is the coordination of a pair of stations to agree and tune their systems to transmit and receive on the same wavelength. One design issue that must be determined before deciding for such protocols is the tunable part of the system.

A scalable optical network can be constructed by taking several WDM links and connecting them with particular wavelength - selective switching subsystems. The signal path through the network is uniquely determined by the wavelength of the signal and the part through which it enters the network. There are actually two types of wavelength switching, one of which dynamically switches signals from one path to another by changing the WDM routing in the network.

The other type wavelength switching is a wavelength conversion, in which the information carried by a signal is transferred from an optical carrier at one wavelength to another.

Our existing networks provide three basic types of transport services:

- $\quad$ circuits - POTS, T-1, SONEY,

- datagram -IP with its best effort service,

- virtual circuits - frame really and ATM.

These are all traditionally electronic domain services. Some or all of such services can now be provides in the optical domain. Fig. 1 shows how the optical network can interface with recent existing services inside an overall broadband network architecture. Network services are provided in the form of layers of various protocols.

A critical factor in the research all-optical networking is the availability of experimental networks or test beds. These large scale implementations represent intensive collaboration between academia, industry and government. A wide range of complementary and independent research projects can be incorporated within the scope of a single test bed program, and in some cases previous stand-alone testbeds are also being linked to provide extended research environments. 


\section{CONCLUSIONS}

This article has briefly summarized the state of the education technology and possible future directions for photonics education. It is clear that the significant engineering issues must be addressed and many systems and management problems must be solved before ubiquitous networking is achieved. As global infrastructures begin to emerge and a host of broadband services appear and deliver economical service to users at offices and homes, the photonic networking will become a serious candidate for wide spread implementation. In conclusion is possible to say, that our students will be in a position to solve these problems.

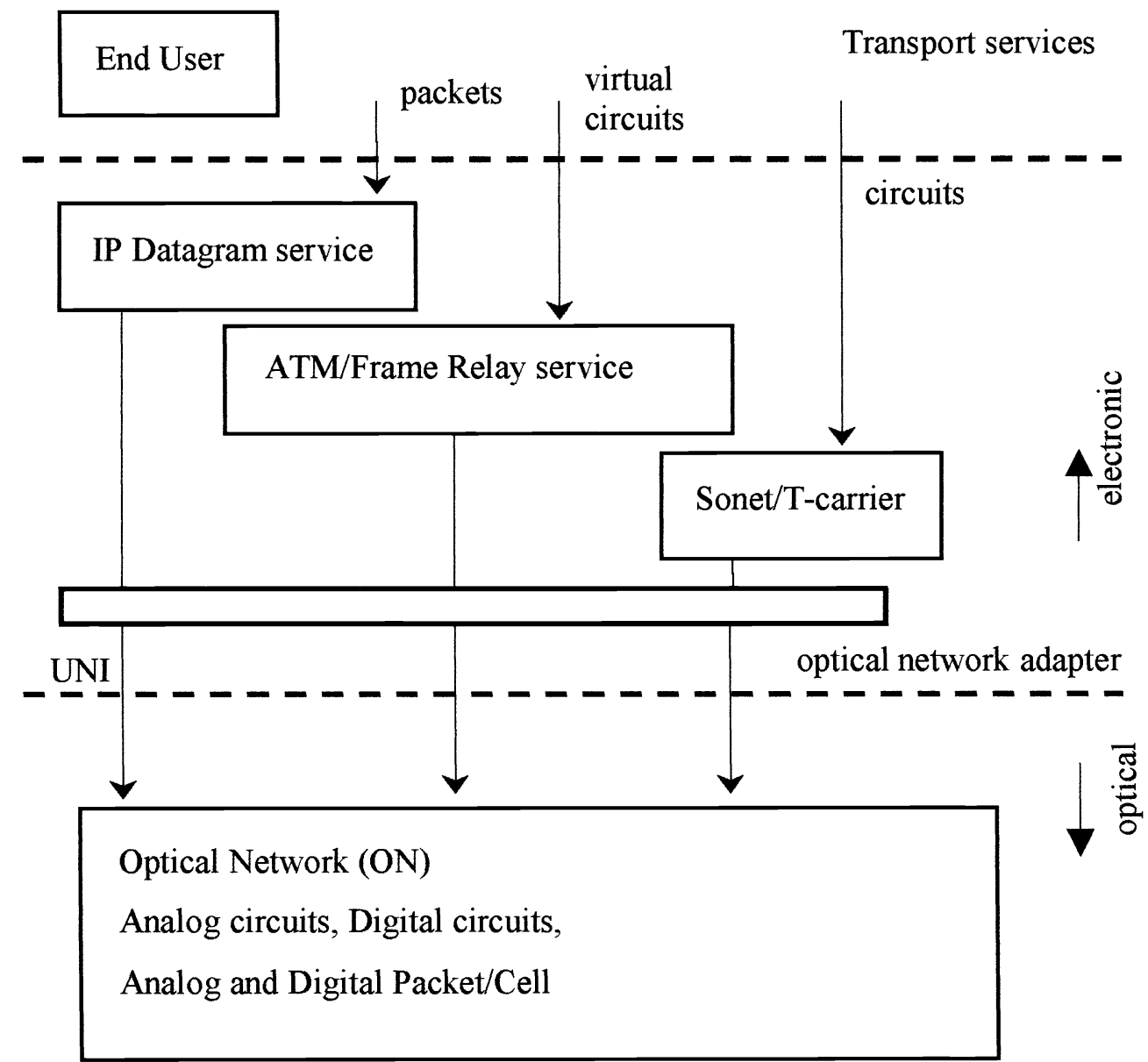

UNI - User Network Interface

Fig. 1

\section{REFERENCES}

[1] P.E.Green - Fiber-Optic Networks, Prentice Hall, New York, 1993

[2] R. Volner, M. Klima, D. Ticha-Photonics, textbook of University in Zilina, 2000 